\title{
Walsh on Causes and Evolution*
}

\author{
Robert Northcottt:
}

Denis Walsh has written a striking new defense in this journal of the statisticalist (i.e., noncausalist) position regarding the forces of evolution. I defend the causalist view against his new objections. I argue that the heart of the issue lies in the nature of nonadditive causation. Detailed consideration of that turns out to defuse Walsh's 'description-dependence' critique of causalism. Nevertheless, the critique does suggest a basis for reconciliation between the two competing views.

1. Introduction. Denis Walsh has written a striking new defense in this journal of the statisticalist position regarding the forces of evolution (Walsh 2007). He rejects the view of those such as Sober (1984), Stephens (2004), and Millstein (2006) that these forces should be seen as causal and thus that they are even aptly labeled 'forces' at all. Instead, following Matthen and Ariew (2002), Walsh, Lewens, and Ariew (2002), and others, he supports viewing selection and drift as mere statistical summaries of evolutionary outcomes. ${ }^{1}$ I offer a response to his arguments and defend the causalist view (a.k.a. the 'dynamical' or 'traditional' view in Walsh's phrasing) against his new criticisms.

Walsh distinguishes between individual-level causalists and populationlevel ones ('macrocausalists'). He argues convincingly against the former. Accordingly, from hereon I consider only the macrocausalist alternative

*Received December 2009; revised December 2009.

$\dagger$ To contact the author, please write to: Department of Philosophy, 599 Lucas Hall, One University Boulevard, University of Missouri, St. Louis, MO 63121; e-mail: northcottr@umsl.edu.

\$This article began life as a commentary on Walsh's article at the Kline workshop in philosophy of biology that took place in September 2007 at the University of MissouriColumbia. I thank Andre Ariew for organizing that workshop and Andre and the other participants for stimulating discussion at it, some of which I have cited here. I also benefited from discussing Walsh's article at the St. Louis philosophy of science reading group and from several comments by an anonymous referee for this journal. Finally, I would especially like to thank Denis Walsh himself.

1. I follow Walsh in considering explicitly only selection and drift among the evolutionary forces, although analogous arguments apply to others also.

Philosophy of Science, 77 (July 2010) pp. 457-467. 0031-8248/2010/7703-0002\$10.00

Copyright 2010 by the Philosophy of Science Association. All rights reserved. 
to statisticalism. Regarding that comparison, Walsh comments: "The only apparent difference [between the two positions] is that the dynamical interpretation applies the term 'cause' to the statistical parameters $<\operatorname{Pr}(p)$, $\operatorname{Pr}(q)>$ and $n$, whereas the statistical interpretation withholds that particular honorific" (2007, 292). I fully agree with this summary of the debate. I would emphasize one implication of it - that the crux of dispute therefore concerns not so much issues in biology but rather issues in the theory of causation and explanation. It is the latter that will be my own focus here.

2. The Case for Macrocausalism. Walsh himself lists some of the reasons why, as it were, it would be desirable to deem these statistical parameters to be causes if we possibly could. As he remarks, they

appear to qualify as genuine causes on most of the prominent and plausible criteria for individuating causes (Millstein 2006). They are (evidently) difference makers on which experimental intervention is possible (Reisman and Forber 2005). Changes in population structure are (allegedly) counterfactually dependent on them. They also count as causes on the chance-raising criterion of causation. Selection, the inequality of $\operatorname{Pr}(p)$ and $\operatorname{Pr}(q)$, systematically raises the chances of a particular kind of outcome, that is, the preponderance of one trait over the other. Drift (sample size) systematically raises the chances of divergence from, or convergence upon, the outcome expected given the values of $\operatorname{Pr}(p)$ and $\operatorname{Pr}(q)$ (Sober and Shapiro 2007). These parameters probabilify their respective outcomes and hence explain them. $(2007,292)$

Thus, as Walsh accepts, the onus is on him to say why they are not causes. This is particularly so because, according to the theories of causation most commonly applied to special sciences (e.g., Woodward 2003), selection and drift both satisfy sufficient conditions for causation. Thus, either a different theory of causation must be offered and defended or else the argument already ends here. ${ }^{2}$

2. One alternative line of counterargument (Walsh, personal communication) is to dispute whether selection and drift really do satisfy these sufficient conditions. In particular, this objection runs, it is not clear whether it is possible, even in principle, to manipulate selection and drift separately, i.e., roughly speaking, to intervene on one without at the same time altering the other. This is relevant because in Woodward's theory a necessary condition for causation is precisely the ability to intervene 'cleanly' in this way, so if that were not possible it would argue against regarding selection and drift as causes. In response, I have two points. First, more work is required to establish the premise, for some maintain that selection and drift can be intervened on cleanly (Reisman and Forber 2005). Second, one criticism of Woodward's theory has been 
A further consideration also tells in favor of the causalist view. That concerns the nature of explanations in population genetics. By his own assumption, Walsh's 'statistical explanations' are not causal, yet they do not appear to be covering-law or unification explanations either. ${ }^{3}$ Therefore, some new notion of explanation would have to be established instead. Alternatively, perhaps it might be denied, against overwhelming scientific practice, that the explanations of population genetics really are explanations, but then some further defense would be required of that radical claim. On the causalist view, in contrast, the problem is immediately resolved by deeming these explanations examples of causal explanation.

For these reasons, I endorse viewing selection and drift as causes and, in particular, as population-level causes. Yet, the above considerations notwithstanding, of course the heart of Walsh's article is to raise some interesting new problems for this causalist view. In the rest of this article, I explain why I think these new problems are not really problems after all. But in the course of that, it will become clear that it is of independent interest to see why not and that Walsh's objections do oblige causalists to refine their position.

3. The Pharaoh Example. It is easy to get confused in probabilistic examples, especially when focusing on nonadditive causes. For this reason, rather than turning to Walsh's own case immediately, I start by getting straight on an analogous but simpler case instead.

Imagine 100 people pushing a giant rock, say ancient Egyptian forced laborers working on a pyramid construction under the gaze of the watching pharaoh. Let the mean force exerted by each laborer be X. To begin with, assume that, being forces, the individual laborers' pushes compose additively, so that the total forward force on the rock is just the sum of the 100 individual pushes (i.e., $100 \mathrm{X}) .{ }^{4} \mathrm{Next}$, suppose for purposes of analysis that we divide these 100 laborers into 10 groups of 10 . Each of

that it is too strict precisely because it makes such clean interventions necessary. The claim (e.g., Cartwright 2007) is that many bona fide causes do not satisfy this condition. If the criticism is right, that would undercut the anticausalist argument here because it suggests that a better theory of causation would not make such clean interventions necessary. That is, on this rival view, the difficulty in intervening on selection and drift cleanly would be not a strike against regarding those factors as causes but instead a strike against the insistence that such clean interventions are necessary in the first place. Thus, the issue is specific to only one (albeit influential) theory of causation. Other theories are less strict.

3. If they were covering-law ones, then presumably the 'law' involved would be the mathematical derivation. But the orthodox view is that such covering laws should be contingent, not necessary.

4. I am assuming that all the pushes are in the same forward direction. 
these subgroups will have a different mean pushing force, label them as $\mathrm{X}_{1}, \mathrm{X}_{2}, \ldots \mathrm{X}_{10}$, respectively. What can we say about the relation between these subgroups' means $X_{i}$ and the total group's mean $X$ ?

Notice, first, one thing that we cannot say:

1. All the $X_{i}$ are equal to $X$.

This is clearly false (in general). So even though the overall group of 100, of course, is just the collection of the 10 subgroups, and even though the pushes are clearly causes (of the rock's motion, of the laborers' sweat, etc.), it does not follow that the average causal strengths in each subgroup will equal the average causal strength in the group as a whole. One thing, however, that we can still assert is:

2. The mean of the $\mathrm{X}_{i}$ must equal $\mathrm{X}^{5}{ }^{5}$

Now tweak the example to make it a better analog of the situation with selection and drift. In particular, that means tweaking it so that the causes are nonadditive. For instance, suppose the relevant cause is the variance of the individual pushes' force-imagine that the pharaoh is greatly impressed by uniformity of laborer effort, and focus on the laborers' pushes as causes of his disapproval. Let the variance of the overall group of 100 pushes be Y, measuring how much the overall group annoys the pharaoh. Let each individual subgroup's respective variances be $\mathrm{Y}_{1}, \mathrm{Y}_{2}, \ldots \mathrm{Y}_{10}$, corresponding to how much each subgroup in isolation annoys the pharaoh.

What now can we say about the relation between the subgroups' $Y_{i}$ and the overall group's Y? Nothing simple. In particular, we will not be able to make either of the following statements:

1. All the $Y_{i}$ are equal to $Y$.

2. The mean of the $Y_{i}$ equals $Y$.

We were able to assert item 2 in the additive case, but (in general) we can no longer, even if the mean is appropriately weighted. Yet we are still talking about causes. How much did each subgroup in isolation cause the pharaoh to be annoyed, and how much did the group as a whole?

If the pharaoh considered each subgroup in turn, his cumulative total annoyance would be different from his level of annoyance if he had simply considered the entire population as a whole. It is precisely this difference that establishes nonadditivity here. Clearly causes in general may be non-

5. For asymmetric group divisions, even this claim would need to be qualified. For example, if we split the group into two subgroups of 30 and 70, then only the appropriately weighted mean of the subgroup means would equal the overall population mean. 
additive, and in particular it is not in dispute that selection and drift are. The above two facts about causal strength in groups and subgroups will be true of the strengths of selection's and drift's contributions to evolution. In particular, it does not follow that just because selection and drift are causes, their strengths in each subsample of a group need bear any simple relation to their strengths in the group as a whole. It is perfectly noncontradictory for drift, for instance, to be strong in each subgroup while simultaneously being weak in the group as a whole - even though the group as a whole is just the sum of those subgroups. That is the lesson of the pharaoh example. ${ }^{6}$

4. Walsh's Coin-Tossing Example. A central illustration that Walsh uses to make his case is a coin-tossing example. Imagine 100 tosses of a fair coin. The variance around a $50: 50$ heads-tails result is small. But now consider an exhaustive partition of this sample into 10 subsets of 10 tosses. Within each subset, the variance around a $5: 5$ result is high. By analogy, drift is high within each subpopulation but low in the population as a whole. (See Walsh [2007], 293-300, for details and a numerical illustration.) Walsh also adapts this example to the case of selection, but the points I want to make can be seen using just the case of drift.

All agree that the degree of drift in each subpopulation of 10 tosses is much higher than the degree of drift in the population as a whole. Walsh argues that because drift is strong in each subpopulation, the causalist view "is committed to the claim that drift-the-cause is strong in the aggregate of 10 sequences of 10 tosses" too $(2007,296)$. But given nonadditivity, this does not follow. The causalist view need have no commitment to the implication that because drift's strength is high in each subgroup, its strength is high in the population as a whole, and the pharaoh example illustrated why. For in just the same way, in the pharaoh case the variance of the workers' efforts could be high in each subgroup and low in the group as a whole, perfectly compatibly with being a cause.

Elliott Sober (personal communication) has suggested an example from biology that illustrates a similar point. In Sober and Wilson's (1998) account of group selection, they discuss how ('genes for') altruism may fare less well than ('genes for') selfishness within any group but that groups with high proportions of altruism may do better as groups than those with high proportions of selfishness. If conditions are set up right, the proaltruism intergroup causal process may overcome the antialtruism in-

6. This analysis is underpinned by the analysis of causal strengths in general. One feature that becomes apparent from that is the acute context specificity of any cause's strength, especially in cases of nonadditive interaction (Northcott 2006). The pharaoh example, and selection and drift, are merely particular instances of this general fact. 
tragroup processes, and the overall frequency of altruism in the population therefore increases. The relevance here is this: in such circumstances, the strength of selection for altruism relative to selfishness may be negative within each group considered in isolation ('subgroup description'), even while simultaneously being positive within the population as a whole ('whole-population description') and even though the population as a whole obviously is just the collection of individual groups. There is no logical inconsistency here hence no contradiction. Formally, the strengths of selection for altruism compose nonadditively across groups, in this case because of the higher-level intergroup causal process.

5. Causation versus Explanation. Walsh comments further on drift's being stronger in the subpopulations than in the population as a whole: "But these are not two populations; they are different ways of describing the same population. . . . The dynamical interpretation is committed to the conclusion that, in this population, the force (or causal process) of drift exists, and is both strong and not strong" $(2007,296)$. According to Walsh, as a result the causalist faces a dilemma - either to accept a contradiction or else arbitrarily to privilege one particular description at the cost of loss of explanatory power. But in light of the preceding sections, I dispute the first horn of this dilemma. In particular, contrary to Walsh, the causalist view does not face any contradiction. The root of the issue lies in Walsh's earlier stipulation that causes should obey a condition of description independence. If accepted, this condition would indeed imply a contradiction, just as he claims. So we must turn to that condition explicitly. (Again, notice that matters turn on an issue in the general theory of causation rather than on any in biology.) Walsh states it as follows: "If $x$ causes $y$, then this relation holds no matter how $x$ and $y$ are described" (292-93).

The view that causation is description independent has a long history (e.g., Davidson 1980). But it is not without opponents. For instance, the influential recent Bayes net literature (e.g., Pearl 2000; Spirtes, Glymour, and Scheines 2000) and related theories of causation (Woodward 2003; Hitchcock 2007) in effect endorse an objective but relational view of causation. As a result, if $x$ and $y$ are interpreted as actual events or actual values of a variable, then whether $x$ causes $y$ can be sensitive to description. In Woodward's scheme, for instance, description dependence enters because different descriptions can serve to pick out different contrast levels of a variable; reality only fixes the variable's actual level (2003, 146; see also 67-68, 210-15). A recent strand in the metaphysics literature has promoted a related contrastive view of causation (Maslen 2004; Schaffer 2005; Northcott 2008). According to this view, Lewis's (1973) counterfactual scheme is also best interpreted as contrastive because of problems 
otherwise with evaluating the relevant counterfactuals correctly. Thus, it is controversial for Walsh to assert without argument that causal relations are description independent. For if the above approaches to causation are right, then the claim is incorrect, and Walsh's critique here of the causalist view fails.

But in any case, for the purposes of the causalist-statisticalist debate, I do not think we need take a stand on whether causation is or is not description independent. Rather, all the causalist needs is the far less controversial claim that causal explanation is not description independent. And this was explicitly conceded by Davidson. Other prominent advocates include Dretske (1972), Van Fraassen (1980), Garfinkel (1981), Achinstein (1983), Hitchcock (1996), and Woodward (2003). It is fair to say that it is the current mainstream view in the literature. But then it follows that the description dependence of selection's and drift's strengths is no argument against viewing them as causally explanatory. That is, we are still free to regard them as causally explaining evolutionary outcomes, just as the causalist view has always insisted. Whether we regard their impacts as causal strengths, or instead merely as 'explanatory strengths' or 'degrees of causal relevance', does not seem to be the central issue here. Either way, we have a causal explanation of evolutionary outcomes and are entitled to ascribe causal (or explanatory) responsibility for these outcomes to selection or drift.

Notice also that Walsh himself is in effect committed to the adequacy in this context of causal explanation as opposed to causation. In particular, when arguing against Bouchard and Rosenberg (2004), he defends only the explanatory autonomy of the macrorealm and hence the inadequacy of microcausal explanations of evolutionary outcomes. It would have been far more controversial to assert also the causal autonomy of the macrorealm (as of course Walsh recognizes - personal communication). But by exactly the same token, it is sufficient here for the macrocausalist if selection and drift can be said to provide merely causal explanations as opposed to causes. For if mere causal explanation is not sufficient, then neither was it sufficient to motivate the statisticalist over the microcausalist view. In other words, Walsh faces a dilemma: either insist on causation rather than causal explanation, at the expense of requiring more work to repel the microcausalists, or else be happy with mere causal explanation, at the expense of giving up the description-dependence objection against the macrocausalists (or else give an argument for why, against the mainstream view in the literature, even causal explanation is not description dependent).

6. How Many Explananda? In light of causal explanation's description dependence, it should not be surprising that drift and selection are as- 
signed different causal/explanatory strengths in each subgroup than they are in the group as a whole - even though that overall group is just the collection of subgroups. That is because any explanatory strength is explanandum specific, and these different situations constitute different explananda.

For example, Walsh notes that seeing the coin-tossing example as 10 independent miniexperiments "offers no explanation of this tendency for error effects to cancel out [when the 10 minisamples are aggregated]" $(2007,296)$. Later in the same paragraph, he notes that seeing the example as, by contrast, one big 100-flip experiment would now "offer no account of why the errors in the subsamples of 10 flips are regularly so large." But the quoted desiderata are clearly two separate explananda, namely, the extent of drift in the large group, on one hand, and then the extent of drift in the small groups, on the other. So we should indeed demand two different (causal) explanations of these two explananda and thus two different explanatory weights for drift, which is exactly what the causalist interpretation offers. ${ }^{7}$

Of course, there is only one actual state of affairs, and it is purely a matter of choice whether we describe it as 10 subgroups or as one big overall group. But in general, just appealing to the actual state of affairs is insufficient to specify an explanandum fully - that is the key implication of causal explanation's description dependence. In particular, depending on how we choose to describe an actual state of affairs, we can generate any of a number of possible explananda and hence any of a number of possible strengths for drift, selection, and so on. ${ }^{8}$ Again, there is no contradiction here.

7. Statistical Truth Makers. One prostatisticalist intuition is that, as it were, the hearts of drift and selection explanations are statistical, not causal. For instance, is not the phenomenon we call drift merely an ex-

7. It is true that a yet further explanandum is why drift has different explanatory weights in the subgroup and whole-population cases. No doubt statistical considerations may be relevant to that, perhaps in the role of truth makers in some intuitive sense (see sec. 7). The point here is only that the fact of these different explanatory weights does not imply any contradiction for the causalist.

8. For example, as previously noted, one popular formulation of description dependence here is via contrast classes. According to that view, causal explanation does not take the general form ' $c$ explains $e$ ' but rather the form ' $c$-rather-than- $c^{*}$ explains $e$ rather-than- $e^{*}$, where $c^{*}$ and $e^{*}$ are contrast classes associated with $c$ and $e$, respectively. It follows that merely citing the actual event $e$ underdetermines the explanandum because it leaves $e^{*}$ unspecified. Therefore, choice of explanandum becomes description dependent - on the contrastive view, different descriptions serve, implicitly or explicitly, to specify different choices of $e^{*}$. 
pression of the purely statistical relation between population size and variance? To identify a drift explanation as causal, so the objection runs, therefore seems to mischaracterize what the real truth makers of that explanation are.

In reply, many of the theories of causation most relevant to biology, such as Woodward's, are silent as to causal claims' ultimate truth makers. On Woodward's scheme, for instance, all that is required is an appropriate invariance relation between the cause and the effect variables. Here that boils down to requiring that manipulation of population size impacts on variance in the prescribed way-which, as Walsh himself concedes, it does (although see n. 2).

Generally, identifying the truth makers of causal claims is a complex issue in metaphysics, full discussion of which is beyond the scope of this article. But one important point is relevant here, namely, that a relation may still be causal even if its truth makers are 'statistical' in some intuitive sense. For example, imagine being in a warm room in winter and opening the window. This action causes the temperature in the room to fall, as cold air from the outside blows in. The relation between opening the window and the fall in room temperature seems to be paradigmatically causal. Yet its truth maker is statistical-the room temperature drops merely because it is with overwhelming probability that the gas molecules inside and outside the room mingle through the open window in such a way as to yield that result. In exactly the same way, the relation between population size and variance (i.e., drift) may be causal even if its truth maker is statistical. ${ }^{9}$

8. Reconciliation? I have defended the macrocausalist view against Walsh's criticisms of it, but I would like to close on a more positive note. In particular, I think the macrocausalist must accommodate the description dependence that Walsh notes and the nonadditivity that generates it. ${ }^{10}$ Depending on metaphysical taste, this in turn requires accepting either

9. Some might have qualms that the window opening is a case of causation by omission, and thus strictly speaking a case of explanation rather than causation. In particular, opening the window merely allows the air molecules to flow through; it contributes no relevant causal 'oomph' of its own. But we may easily substitute an alternative example to make the same point, say that lighting a fire causes the far end of a room to heat up. Again the truth maker is 'statistical', but now our action is a positive cause. In any case, as argued earlier, for this article's purposes even causal explanation is sufficient.

10. Any allegiance to Woodward's theory of causation implicitly commits one to such description dependence already. But I do not think this is any special embarrassmentnonadditive causation is the norm in special sciences, so evolutionary theory is hardly alone in this respect. 
that causation is description dependent or else that drift and selection are merely causal explanatory as opposed to causal.

One metaphor, popular with causalists since Sober (1984), compares drift and selection to Newtonian forces. But, by definition, Newtonian forces compose additively. ${ }^{11}$ Thus, the metaphor is inevitably inadequate in the face of the description dependence highlighted by Walsh.

However, once the above points are taken on board, it seems to me that a thus-elaborated version of causalism will have accommodated many of statisticalism's main worries. In particular, the causalist will have embraced the critique of the microcausalist view of evolutionary forces, the critique of attributing causal status to drift (or selection)-the-product as opposed to drift-the-process (to use the phrasing of Millstein [2002]), the statistical element in evolutionary explanations, and the description dependence highlighted by Walsh. And in return for dropping their opposition, the statisticalists would thereby be freed to accept much-needed help from causalism against the problems of section 2 .

\section{REFERENCES}

Achinstein, Peter. 1983. The Nature of Explanation. Oxford: Oxford University Press.

Bouchard, Frederic, and Alexander Rosenberg. 2004. "Fitness, Probability and the Principles of Natural Selection." British Journal for the Philosophy of Science 55:693-712.

Cartwright, Nancy. 2007. Hunting Causes and Using Them: Approaches in Philosophy and Economics. Cambridge: Cambridge University Press.

Davidson, Donald. 1980. "Causal Relations." In Essays on Actions and Events, 149-62. Oxford: Clarendon.

Dretske, Fred. 1972. "Contrastive Statements." Philosophical Review 81 (4): 411-37.

Garfinkel, Alan. 1981. Forms of Explanation. New Haven, CT: Yale University Press.

Hitchcock, Christopher. 1996. "The Role of Contrast in Causal and Explanatory Claims." Synthese 107:395-419.

- 2007. "Prevention, Preemption, and the Principle of Sufficient Reason." Philosophical Review 116:495-532.

Lewis, David. 1973. "Causation.” Journal of Philosophy 70:556-67.

Maslen, Cei. 2004. "Causes, Contrasts and the Nontransitivity of Causation." In Causation and Counterfactuals, ed. John Collins, Ned Hall, and L. A. Paul, 341-58. Cambridge, MA: MIT Press.

Matthen, Mohan, and Andre Ariew. 2002. "Two Ways of Thinking about Fitness and Natural Selection." Journal of Philosophy 119:55-83.

Millstein, Roberta. 2002. "Are Random Drift and Natural Selection Conceptually Distinct?" Biology and Philosophy 17:33-53.

- 2006. "Natural Selection as a Population-Level Causal Process." British Journal for the Philosophy of Science 57:627-53.

Northcott, Robert. 2006. "Causal Efficacy and the Analysis of Variance." Biology and Philosophy 21 (2): 253-76.

. 2008. "Causation and Contrast Classes." Philosophical Studies 39:111-23.

Pearl, Judea. 2000. Causality. New York: Cambridge University Press.

11. Sober (1984) recognizes this. Newtonian forces are also nonprobabilistic, thus rendering them further disanalogous to drift and selection. See Stephens (2004) for discussion of other respects in which the metaphor is and is not apt. 
Reisman, Kenneth, and Patrick Forber. 2005. "Manipulation and the Causes of Evolution." Philosophy of Science 72 (Proceedings): 1113-23.

Schaffer, Jonathan. 2005. "Contrastive Causation." Philosophical Review 114 (3): 297-328. Sober, Elliott. 1984. The Nature of Selection. Cambridge, MA: MIT Press.

Sober, Elliott, and Lawrence Shapiro. 2007. "Epiphenomenalism-Some Do's and Don'ts." In Studies in Causality: Historical and Contemporary, ed. G. Wolters and Peter Machamer, 235-64. Pittsburgh: University of Pittsburgh Press.

Sober, Elliott, and David Sloan Wilson. 1998. Unto Others: The Evolution and Psychology of Unselfish Behavior. Cambridge, MA: Harvard University Press.

Spirtes, Peter, Clark Glymour, and Richard Scheines. 2000. Causation, Prediction, and Search. 2nd ed. Cambridge, MA: MIT Press.

Stephens, Christopher. 2004. "Selection, Drift and the 'Forces' of Evolution." Philosophy of Science 70:280-301.

Van Fraassen, Bas. 1980. The Scientific Image. Oxford: Oxford University Press.

Walsh, Denis. 2007. "The Pomp of Superfluous Causes: The Interpretation of Evolutionary Theory." Philosophy of Science 74:281-303.

Walsh, Denis, Tim Lewens, and Andre Ariew. 2002. "The Trials of Life: Natural Selection and Drift." Philosophy of Science 69:452-73.

Woodward, James. 2003. Making Things Happen: A Theory of Causal Explanation. Oxford: Oxford University Press. 